Title:

Authors:

Affiliation 1:

Affiliation 2:

Affiliation 3:

Address:

Colloquium:

Total Length:

(Method: M1)

\section{Front shock behavior of stable curved detonation waves in rectangular-cross-section curved channels}

\author{
HISAHIRO NAKAYAMA ${ }^{1}$, JIRO KASAHARA ${ }^{1}$, AKIKO MATSUO ${ }^{2}$, \\ IKKOH FUNAKI ${ }^{3}$
}

Department of Engineering Mechanics and Energy, University of Tsukuba 1-1-1 Tennodai, Tsukuba, Ibraki 305-8573, Japan

Department of Mechanical Engineering, Keio University

3-14-1 Hiyoshi, Kouhoku-ku, Yokohama, Kanagawa 223-8522, Japan

Institute of Space and Astronautical Science, Japan Aerospace Exploration Agency

3-1-1 Yoshinodai, Chuo-ku, Sagamihara, Kanagawa 252-5210, Japan

JIRO KASAHARA, Department of Engineering Mechanics and Energy,

University of Tsukuba

1-1-1 Tennodai, Tsukuba, Ibaraki 305-8573, Japan

E-mail: kasahara@kz.tsukuba.ac.jp

Fax: 029-853-5267

DETONATIONS, EXPLOSIONS AND SUPERSONIC COMBUSTION

including pulse-detonation and scramjet engines

6197 words

$\begin{array}{ll}\text { Main text: } & 2568 \text { words } \\ \text { Nomenclature: } & 113 \text { words } \\ \text { References: } & 340 \text { words } \\ \text { Tables: } & 99 \text { words } \\ \text { Figures: } & 3077 \text { words }\end{array}$




\begin{abstract}
The propagation of curved detonation waves of gaseous explosives stabilized in rectangular-cross-section curved channels is investigated. Three types of stoichiometric test gases, $\mathrm{C}_{2} \mathrm{H}_{4}+3 \mathrm{O}_{2}, 2 \mathrm{H}_{2}+\mathrm{O}_{2}$, and $2 \mathrm{C}_{2} \mathrm{H}_{2}+5 \mathrm{O}_{2}+7 \mathrm{Ar}$, are evaluated. The ratio of the inner radius of the curved channel $\left(r_{\mathrm{i}}\right)$ to the normal detonation cell width $(\lambda)$ is an important factor in stabilizing curved detonation waves. The lower boundary of stabilization is around $r_{\mathrm{i}} / \lambda=23$, regardless of the test gas. The stabilized curved detonation waves eventually attain a specific curved shape as they propagate through the curved channels. The specific curved shapes of stabilized curved detonation waves are approximately formulated, and the normal detonation velocity $\left(D_{\mathrm{n}}\right)$ - curvature $(\kappa)$ relations are evaluated. The $D_{\mathrm{n}}$ nondimensionalized by the Chapman-Jouguet (CJ) detonation velocity $\left(D_{\mathrm{CJ}}\right)$ is a function of the $\kappa$ nondimensionalized by $\lambda$. The $D_{\mathrm{n}} / D_{\mathrm{CJ}}-\lambda \kappa$ relation does not depend on the type of test gas. The propagation behavior of the stabilized curved detonation waves is controlled by the $D_{\mathrm{n}} / D_{\mathrm{CJ}}-\lambda \kappa$ relation. Due to this propagation characteristic, the fully-developed, stabilized curved detonation waves propagate through the curved channels while maintaining a specific curved shape with a constant angular velocity. Self-similarity is seen in the front shock shapes of the stabilized curved detonation waves with the same $r_{\mathrm{i}} / \lambda$, regardless of the curved channel and test gas.
\end{abstract}

\title{
Keywords
}

Curved detonation wave, Curvature, Normal detonation velocity, Cell width, Rotating detonation engine

\section{Nomenclature}

D: $\quad$ detonation velocity

m: $\quad$ exponent in Eq. (3) 
Proceedings of the Combustion Institute 34 (2013) 1939-1947

H. Nakayama, J. Kasahara, A. Matsuo, I. Funaki

p: $\quad$ pressure

$r$ distance from the polar coordinate origin to an arbitrary point on the detonation wave

t: $\quad$ time

\section{Greek symbols}

$\phi: \quad$ angular difference between rotational and tangential directions at an arbitrary point on the detonation

wave

$\kappa$ detonation wave curvature

$\lambda: \quad$ normal detonation cell width

$\theta$ angle from the initial line to an arbitrary point on the detonation wave

$\tau$. nondimensional time

$\omega$ : $\quad$ angular velocity of an arbitrary point on the detonation wave

\section{Subscripts}

0: $\quad$ initial condition

asy: asymptotic value at sufficiently large $r$

CJ: Chapman-Jouguet

$\mathrm{i}: \quad$ inner wall of a curved channel

$\mathrm{n}: \quad$ normal direction

o: $\quad$ outer wall of a curved channel 


\section{Introduction}

Since a rotating detonation engine (RDE) [1-4] utilizes a combustible gas mixture as a propellant and its combustor is annular, a curved detonation wave propagating continuously is seen in its combustor. Therefore, it is important that the propagation of the curved detonation wave should be well understood for the purpose of RDE design.

Many theoretical/experimental studies on steady/quasi-steady curved Zeldovich-von Neumann-Döring (ZND) detonation waves have been performed in the field of condensed-phase explosives [5-12]. However, studies on curved detonation waves of gaseous explosives are scarce, since it is difficult to attain steady curved detonation waves of gaseous explosives. Hence, the propagation characteristics of curved detonation waves of gaseous explosives are not yet sufficiently understood.

Kasahara et al. $[13,14]$ and Maeda et al. $[15,16]$ launched a hypersonic projectile into an explosive gas mixture to generate a steady conical curved detonation wave around the projectile. Although each set of authors mentioned the influence of $\kappa$ on the stability of the conical curved detonation wave, they could not discuss this issue quantitatively. Kudo et al. [17] demonstrated the steady propagation of a curved detonation wave of $\mathrm{C}_{2} \mathrm{H}_{4}+3 \mathrm{O}_{2}$ stabilized in a rectangular-cross-section curved channel with constant inner/outer radii of curvature, however, they could not obtain the $D_{n}-\kappa$ relation of $\mathrm{C}_{2} \mathrm{H}_{4}+3 \mathrm{O}_{2}$. Nakayama et al. [18] used the same gas mixture and simultaneously visualized the front shock and cell structure of a curved detonation wave stabilized in a rectangular-cross-section curved channel with very-shallow depth by employing multi-frame short-time open-shutter photography (MSOP). They showed that the smooth generation of new detonation cells within the enlarged cells stabilizes the propagation of a curved detonation wave and keeps its curved shape smooth [18]. They also formulated a good approximation of the curved shape and showed quantitatively the $D_{\mathrm{n}}-\kappa$ relation of $\mathrm{C}_{2} \mathrm{H}_{4}+3 \mathrm{O}_{2}$ for the first time [18]. However, their 
Proceedings of the Combustion Institute 34 (2013) 1939-1947

H. Nakayama, J. Kasahara, A. Matsuo, I. Funaki

quantitative examination on the relation was performed at a limited high $p_{0}$ (i.e., small $\lambda$ ) since their experimental

results were affected by momentum loss due to very-shallow channel depth. Although Nakayama et al. [19]

subsequently re-examined the $D_{\mathrm{n}}-\kappa$ relation by using curved channels with sufficient depth to avoid momentum

loss and showed the stabilization condition of curved detonation waves propagating through them, the propagation

of curved detonation waves of gaseous explosives was not elucidated sufficiently since only the one gas mixture,

$\mathrm{C}_{2} \mathrm{H}_{4}+3 \mathrm{O}_{2}$, was used in their study.

Therefore, three types of gas mixture are evaluated under a wide range of initial pressure to investigate extensively the propagation characteristics of a stabilized curved detonation wave in the present study. Rectangular-cross-section curved channels with constant inner/outer radii of curvature are used to stabilize the curved detonation waves like in the previous studies [17-19]. The stabilized curved detonation waves of these gas mixtures are examined to evaluate their $D_{\mathrm{n}}-\kappa$ relations. The front shock evolution of stabilized curved detonation waves is reconstructed by using these $D_{\mathrm{n}}-\kappa$ relations and the universal propagation characteristics of the stabilized curved detonation waves are elucidated.

\section{Geometric front shock shape of stabilized curved detonation wave in a curved channel}

As shown in Fig. 1, the center of the inner/outer radii of curvature of a rectangular-cross-section curved channel is defined as the origin, and the boundary between the curved and straight sections of the curved channel is defined as the initial line in a two-dimensional polar coordinate system. It is assumed that a stabilized curved detonation wave propagates through the curved channel while maintaining a specific curved shape and that $\omega$ is time-unvarying as well as constant everywhere on the curved detonation wave. Then, the following differential equation can be established at an arbitrary point $\mathrm{P}(r, \theta)$ on the curved detonation wave $[18,19]$ : 


$$
\frac{\mathrm{d} \theta}{\mathrm{d} r}=-\frac{\sqrt{(r \omega)^{2}-D_{\mathrm{n}}^{2}}}{D_{\mathrm{n}} r},
$$

where $\omega=D_{\mathrm{n}, \mathrm{i}} / r_{\mathrm{i}}$. If $D_{\mathrm{n}}$ is given as a function of $r$, the following relation between $r$ and $\theta$, which gives the front shock shape of the curved detonation wave, is derived by integrating Eq. (1) $[18,19]$ :

$$
\theta-\theta_{\mathrm{i}}=-\int_{r_{\mathrm{i}}}^{r_{\mathrm{o}}} \frac{\sqrt{(r \omega)^{2}-D_{\mathrm{n}}^{2}}}{D_{\mathrm{n}} r} \mathrm{~d} r
$$

The following formula that approximates a $D_{\mathrm{n}}$ distribution of the curved detonation wave are employed $[18,19]$ :

$$
D_{\mathrm{n}}=D_{\mathrm{n}, \mathrm{asy}}-\left(D_{\mathrm{n}, \mathrm{asy}}-D_{\mathrm{n}, \mathrm{i}}\right)\left(r / r_{\mathrm{i}}\right)^{-m},
$$

which is applicable only when the reflection over the outer wall is regular. In Eq. (3), $D_{\mathrm{n}}$ increases from $D_{\mathrm{n}, \mathrm{i}}$ to $D_{\mathrm{n}, \text { asy }}$ asymptotically with increasing $r$, and the constant $m$ gives the increasing rate of $D_{\mathrm{n}}$. Generally, the value of $D_{\mathrm{n}, \text { asy }}$ is neary equal to that of $D_{\mathrm{CJ}}$.

As shown in Fig. 1, the front shock shape of the curved detonation wave is divided into fifteen parts at a regular interval in the direction of $r$. The coordinate values of the division points are picked up, and the front shock shape is defined. The $\omega$ values of each division point are determined by observing the moment-to-moment alternation of their positions. The values of $D_{\mathrm{n}, \text { asy }}$ and $m$ are determined by the trial-and-error technique in such a way that the residual sum of squares between the reconstructed front shock shape of the curved detonation wave using Eqs. and (3) and the coordinate values of the division points acquired experimentally becomes the smallest [19].

\section{Experiment}

Figure 2 shows the schematics of the curved channels. Five types of curved channel with different inner radii of curvature are used. The width and depth of these curved channels are $20 \mathrm{~mm}$ and $16 \mathrm{~mm}$, respectively. A test gas is filled at a given $p_{0}$ in the observation chamber where a curved channel is installed and ignited by a spark plug. A 
Proceedings of the Combustion Institute 34 (2013) 1939-1947

H. Nakayama, J. Kasahara, A. Matsuo, I. Funaki

deflagration wave transitions to a detonation wave within the Shchelkin spiral section located below the curved

channel, and a planar detonation wave enters the curved channel.

Table 1 shows the experimental conditions. Three types of stoichiometric test gases, $\mathrm{C}_{2} \mathrm{H}_{4}+3 \mathrm{O}_{2}, 2 \mathrm{H}_{2}+\mathrm{O}_{2}$, and $2 \mathrm{C}_{2} \mathrm{H}_{2}+5 \mathrm{O}_{2}+7 \mathrm{Ar}$, are used. The $\lambda$ values are converted from $p_{0}$ by using the relations shown in Table 1 . These relations are obtained by applying the least-squares method to the data of $\lambda$ of these test gases extracted from the Detonation Database of Caltech [20]. The velocity of a planar detonation wave entering a curved channel agrees well with $D_{\mathrm{CJ}}$ calculated using CEA [21]. Hence, $D_{\mathrm{CJ}}$ is used as a reference velocity for the nondimensionalization of $D_{\mathrm{n}}$.

Detonation waves are visualized by the shadowgraph or schlieren methods and recorded by a high-speed video camera (Shimadzu HPV-2) at $2-\mu$ s intervals. The spatial resolution of the photographs taken is approximately 0.3 $\mathrm{mm}$.

\section{Results and discussion}

\subsection{Stabilization condition of curved detonation wave}

The propagation mode that consistently satisfies the relation $D_{\mathrm{n}, \mathrm{i}} / D_{\mathrm{CJ}} \geq 0.8$ is defined as the stable mode, the mode that cannot satisfy the relation $D_{\mathrm{n}, \mathrm{i}} / D_{\mathrm{CJ}} \geq 0.8$ but can consistently satisfy the relation $D_{\mathrm{n}, \mathrm{i}} / D_{\mathrm{CJ}} \geq 0.6$ is defined as the critical mode, and the mode in which $D_{\mathrm{n}, \mathrm{i}} / D_{\mathrm{CJ}}<0.6$ even just once is defined as the unstable mode in the present study $[18,19] . D_{\mathrm{n}, \mathrm{i}}$ is measured at a time interval $(\Delta t)$ equal to or longer than $2 \lambda / D_{\mathrm{CJ}}$, and $\Delta t$ is $2 \mu$ s or $4 \mu \mathrm{s}$.

Figure 3 shows the relations between $r_{\mathrm{i}}$ and $\lambda$ in terms of the propagation mode. The propagation mode of a curved detonation wave in a curved channel transitions to the stable mode from the unstable mode approximately within $14 \leq r_{\mathrm{i}} / \lambda \leq 23$. The lower boundary of stabilization is considered to be about $r_{\mathrm{i}} / \lambda=23$ regardless of the test gas. 
Proceedings of the Combustion Institute 34 (2013) 1939-1947

H. Nakayama, J. Kasahara, A. Matsuo, I. Funaki

The detailed description of the determination method of these boundaries is written in Ref. [18].

Figure 4 is an example of a photograph of a curved detonation wave in the stable mode [19]. This photograph was taken by MSOP [18] using the special curved channel of 1-mm depth. The conditions are $\mathrm{C}_{2} \mathrm{H}_{4}+3 \mathrm{O}_{2}, r_{\mathrm{i}}=20 \mathrm{~mm}$, and $\lambda=0.71 \mathrm{~mm}$. The smooth generation of new detonation cells within the enlarged cells stabilizes the propagation of the curved detonation wave and maintains its smooth curved shape.

\subsection{Approximation of front shock shape}

The previous studies $[18,19]$ have shown that the propagation of a curved detonation wave in the stable mode eventually becomes steady and that the assumption described in Chapter 2 is correct. This characteristic is absolutely necessary to obtain the $D_{\mathrm{n}}-\kappa$ relation of the curved detonation wave. Figure 5 shows an example of the front shock shape of a curved detonation wave in the stable mode approximated by Eqs. (2) and (3). The conditions are $\mathrm{C}_{2} \mathrm{H}_{4}+3 \mathrm{O}_{2}, r_{\mathrm{i}}=20 \mathrm{~mm}$, and $\lambda=0.70 \mathrm{~mm} . D_{\mathrm{n}, \mathrm{asy}}=0.980 D_{\mathrm{CJ}}, m=8.10, D_{\mathrm{n}, \mathrm{i}}=0.87235 D_{\mathrm{CJ}}$, and $\omega=1.0231 \times 10^{5} \mathrm{rad} / \mathrm{s}$ are used for the approximation. The error bars represent the typical possible systematic error of measurement resulting from the spatial resolution of the high-speed video camera. The error bars used in figures that follow Fig. 5 represent the same definition. The approximated front shock shape agrees well with the experimental result.

Figure 6 shows an example of the $D_{n}$ distribution of a curved detonation wave approximated by Eq. (3). The conditions are the same as those in Fig. 5. The approximated distribution of $D_{\mathrm{n}}$ is also in good agreement with the experimental result.

\section{3. $D_{n} / D_{C J}-\lambda$ Krelation}

Since the front shock shapes and $D_{\mathrm{n}}$ distribution of curved detonation waves in the stable mode are 
approximately given by Eqs. (2) and (3), the $D_{\mathrm{n}}-\kappa$ relations can be obtained from the approximation results of curved detonation waves. Figure 7 shows the $D_{\mathrm{n}}-\kappa$ relations of curved detonation waves in the stable mode. The test gas is $\mathrm{C}_{2} \mathrm{H}_{4}+3 \mathrm{O}_{2}$. The values of $D_{\mathrm{n}}$ and $\kappa$ are nondimensionalized by $D_{\mathrm{CJ}}$ and $\lambda$, respectively. The ten cases of curved detonation waves at different $r_{\mathrm{i}}$ and/or $\lambda$ were selected to cover the stable zone shown in Fig. 3a. One can see that $D_{\mathrm{n}} / D_{\mathrm{CJ}}$ is a function of $\lambda \kappa$ regardless of the curved channel and that it decreases with increasing $\lambda \kappa$. The same characteristic is also seen in the $D_{n} / D_{C J}-\lambda \kappa$ relations of $2 \mathrm{H}_{2}+\mathrm{O}_{2}$ and $2 \mathrm{C}_{2} \mathrm{H}_{2}+5 \mathrm{O}_{2}+7 \mathrm{Ar}$.

The $D_{\mathrm{n}} / D_{\mathrm{CJ}}-\lambda \kappa$ relations of all the test gases are compared in Fig. 8. For each test gas, several cases covering each stable zone in Fig. 3 were selected to evaluate their $D_{\mathrm{n}} / D_{\mathrm{CJ}}-\lambda \kappa$ relations. The $D_{\mathrm{n}} / D_{\mathrm{CJ}}-\lambda \kappa$ relations of each test gas are approximated by a cubic function. It is noteworthy that the difference in the $D_{\mathrm{n}} / D_{\mathrm{CJ}}-\lambda \kappa$ relation of each test gas is very small, and, more specifically, the $D_{\mathrm{n}} / D_{\mathrm{CJ}}-\lambda \kappa$ relation does not depend on the type of test gas. Therefore, the average of these $D_{\mathrm{n}} / D_{\mathrm{CJ}}-\lambda \kappa$ relations is defined as the overall $D_{\mathrm{n}} / D_{\mathrm{CJ}}-\lambda \kappa$ relation and approximated by a cubic function of $D_{\mathrm{n}} / D_{\mathrm{CJ}}=\sum \mathrm{a}_{j}(\lambda \kappa)^{j}$, where $j=0-3, \mathrm{a}_{0}=1.0000, \mathrm{a}_{1}=-1.3017, \mathrm{a}_{2}=16.089$, and $\mathrm{a}_{3}=-169.67$.

Figure 9 shows examples of the reconstruction of the front shock behavior of curved detonation waves with the overall $D_{\mathrm{n}} / D_{\mathrm{CJ}}-\lambda \kappa$ relation. The marker particle method [22] is employed to track the front shocks of the curved detonation waves. The boundary conditions are set up with the front shock perpendicular to the inner wall and the reflection being regular over the outer wall. The $\Delta t$ value of the front shocks in the photographs is $2 \mu \mathrm{s}$, and that of the reconstructed front shocks is $4 \mu \mathrm{s}$. In all the cases, the front shock behavior is successfully reconstructed using the overall $D_{\mathrm{n}} / D_{\mathrm{CJ}}-\lambda \kappa$ relation. Hence, once the overall $D_{\mathrm{n}} / D_{\mathrm{CJ}}-\lambda \kappa$ relation is given, Eqs. (2) and (3) are no longer necessary. These results show that the propagation behavior of the curved detonation waves is controlled by a specific $D_{\mathrm{n}} / D_{\mathrm{CJ}}-\lambda$ krelation, therefore detonation shock dynamics (DSD) [5-8] can also be applied to stabilized curved detonation waves of gaseous explosives. 
Since the $\lambda-p_{0}$ relations shown in Table 1 are obtained from the data sets with some errors and scatter, the $D_{\mathrm{n}} / D_{\mathrm{CJ}}-\lambda \kappa$ relations shown in Fig. 8 may be affected by them. However, it's been confirmed that the front shock behavior of stabilized curved detonation waves can be successfully reconstructed with the overall $D_{\mathrm{n}} / D_{\mathrm{CJ}}-\lambda \kappa$ relation also in other conditions as well as in Fig. 9. Hence, one can think that the value of $\lambda$ given by these relations is appropriate and the influence of these errors and scatter on the results of the present study is small.

\subsection{Front shock evolution of curved detonation wave}

Figure 10 shows the front shock evolution of curved detonation waves reconstructed with the overall $D_{\mathrm{n}} / D_{\mathrm{CJ}}-\lambda \kappa$ relation. Since the condition over the outer wall does not affect the propagation of a curved detonation wave as long as the reflection over the outer wall is regular, $r_{\mathrm{o}}$ is assumed to be infinite in this analysis. Two values of $r_{\mathrm{i}} / \lambda, 23$ (lower boundary of stabilization) and 50, are selected. The values of $x, y$, and $t$ are nondimensionalized as $x / r_{\mathrm{i}}, y / r_{\mathrm{i}}$, and $t D_{\mathrm{CJ}} / r_{\mathrm{i}}(\equiv \tau)$, respectively. The $\Delta \tau$ between front shocks is 1 . If the propagation of curved detonation waves in the stable mode is controlled by a specific $D_{\mathrm{n}} / D_{\mathrm{CJ}}-\lambda \kappa$ relation, the front shock shapes of the curved detonation waves with the same $r_{\mathrm{i}} / \lambda$ become congruent on the $x / r_{\mathrm{i}}-y / r_{\mathrm{i}}$ plane at a given $\tau$, regardless of $r_{\mathrm{i}}$ (i.e., the channel type). That is to say, self-similarity is seen in the front shock shapes with the same $r_{\mathrm{i}} / \lambda$. The front shock shapes are obviously determined independently from the type of gas mixture if the specific $D_{\mathrm{n}} / D_{\mathrm{CJ}}-\lambda \kappa$ relation is unique. The constant $\lambda \kappa$ and $D_{\mathrm{n}} / D_{\mathrm{CJ}}$ lines become concentric as the curved detonation waves propagate. This means the curved detonation waves eventually attain a specific curved shape and their propagation becomes steady. The curved shapes develop fully within shorter $\tau$ in the curved channel with smaller $r / r_{\mathrm{i}}$ (i.e., a shallower channel). One can predict that the propagation of the curved detonation wave with infinite $r_{\mathrm{i}} / \lambda$ approaches the Huygens construction asymptotically since the $D_{\mathrm{n}} / D_{\mathrm{CJ}}$ deficit and $\lambda \kappa$ decrease with increasing $r_{\mathrm{i}} / \lambda$. 
Proceedings of the Combustion Institute 34 (2013) 1939-1947

H. Nakayama, J. Kasahara, A. Matsuo, I. Funaki

Figure 11 shows the comparison of the front shock shapes of curved detonation waves with different $r_{\mathrm{i}}$. The conditions are $\mathrm{C}_{2} \mathrm{H}_{4}+3 \mathrm{O}_{2}$ and $r_{\mathrm{i}} / \lambda=36.3$. The experimental result also shows that the front shock shapes with the same $r_{\mathrm{i}} / \lambda$ become similar regardless of $r_{\mathrm{i}}$ (i.e., the channel type). The values of $D_{\mathrm{n} \text {,asy }}$ and $m$ determined from the approximation by Eqs. (2) and (3) are $0.965 D_{\mathrm{CJ}}$ and 10.25 for $r_{\mathrm{i}}=20 \mathrm{~mm}, 0.970 D_{\mathrm{CJ}}$ and 11.00 for $r_{\mathrm{i}}=40 \mathrm{~mm}$, and $0.970 D_{\mathrm{CJ}}$ and 10.55 for $r_{\mathrm{i}}=60 \mathrm{~mm}$, respectively. The differences in $D_{\mathrm{n} \text {,asy }} / D_{\mathrm{CJ}}$ and $m$ between the different $r_{\mathrm{i}}$ are very small, and hence this result also supports the self-similarity shown in Fig. 11.

Figure 12 shows the comparison of the front shock shapes of curved detonation waves with different test gases.

The conditions are $r_{\mathrm{i}}=20 \mathrm{~mm}$ and $r_{\mathrm{i}} / \lambda=27.5$. The experimental result also shows that the front shock shapes with the same $r_{\mathrm{i}} / \lambda$ are determined independently from the type of test gas and they are similar. The values of $D_{\mathrm{n}, \text { asy }}$ and $m$ determined from the approximation by Eqs. (2) and (3) are $0.985 D_{\mathrm{CJ}}$ and 7.70 for $\mathrm{C}_{2} \mathrm{H}_{4}+3 \mathrm{O}_{2}, 0.970 D_{\mathrm{CJ}}$ and 0.75 for $2 \mathrm{H}_{2}+\mathrm{O}_{2}$, and $0.980 D_{\mathrm{CJ}}$ and 7.50 for $2 \mathrm{C}_{2} \mathrm{H}_{2}+5 \mathrm{O}_{2}+7 \mathrm{Ar}$, respectively. The differences in $D_{\mathrm{n}, \text { asy }} / D_{\mathrm{CJ}}$ and $m$ between the different test gases are very small, and hence this result also supports the self-similarity between them.

\section{Conclusions}

The propagation of curved detonation waves of gaseous explosives stabilized in rectangular-cross-section curved channels was investigated. The lower boundary of stabilization is around $r_{\mathrm{i}} / \lambda=23$ regardless of the test gas used in the present study. The $D_{\mathrm{n}} / D_{\mathrm{CJ}}$ of a stabilized curved detonation wave is a function of the $\lambda \kappa$, and the $D_{\mathrm{n}} / D_{\mathrm{CJ}}-\lambda \kappa$ relation does not depend on the type of test gas. The front shock evolution of the stabilized curved detonation waves is controlled by the $D_{\mathrm{n}} / D_{\mathrm{CJ}}-\lambda \kappa$ relation. Due to this propagation characteristic, the stabilized curved detonation waves eventually propagate steadily through the curved channels while maintaining a specific curved shape. Self-similarity is seen in the front shock shapes of the stabilized curved detonation waves with the same $r_{\mathrm{i}} / \lambda$ 
Proceedings of the Combustion Institute 34 (2013) 1939-1947

H. Nakayama, J. Kasahara, A. Matsuo, I. Funaki

regardless of the curved channel and test gas.

\section{Acknowledgements}

This work was supported by Grants-in-Aid for Scientific Research (A) (No.20241040) and (B) (No.21360411), MEXT, and by the Research Grant Program, ISAS/JAXA.

\section{References}

[1] M. Hishida, T. Fujiwara, P. Wolański, Shock Waves 19 (2009) 1-10.

[2] J. Kindracki, P. Wolański, Z. Gut, Shock Waves 21 (2011) 75-84.

[3] Y. Eude, D.M. Davidenko, I. Gökalp, F. Falempin, AIAA Paper 2011-2236, 2011.

[4] T. Yamada, A.K. Hayashi, E. Yamada, N. Tsuboi, V.E. Tangirala, T. Fujiwara, Combust. Sci. and Tech. 182 (2010) 1901-1914.

[5] J.B. Bdzil, D.S. Stewart, Phys. Fluids A 1 (7) (1989) 1261-1267.

[6] D.S. Stewart, J.B. Bdzil, Combust. Flame 72 (1988) 311-323.

[7] D.S. Stewart, J. Yao, W.C. Davis, Proc. Combust. Inst. 28 (2000) 619-628.

[8] D.E. Lambert, D.S. Stewart, S. Yoo, B.L. Wescott, J. Fluid Mech. 546 (2006) 227-253.

[9] M. Short, G.J. Sharpe, V. Gorchkov, J.B Bdzil, Proc. Combust. Inst. 30 (2005) 1899-1906.

[10] J. Yao, D.S. Stewart, Combust. Flame 100 (1995) 519-528.

[11] B.L. Wescott, D.S. Stewart, W.C. Davis, J. Appl. Phys. 98, 053514 (2005).

[12] S.I. Jackson, C.B. Kiyanda, M. Short, Proc. Combust. Inst. 33 (2011) 2219-2226.

[13] J. Kasahara, T. Fujiwara, T. Endo, T. Arai, AIAA Journal 39 (8) (2001) 1553-1561. 
Proceedings of the Combustion Institute 34 (2013) 1939-1947

H. Nakayama, J. Kasahara, A. Matsuo, I. Funaki

[14] J. Kasahara, T. Arai, S. Chiba, K. Takazawa, Y. Tanahashi, A. Matsuo, Proc. Combust. Inst. 29 (2002) 2817-2824.

[15] S. Maeda, R. Inada, J. Kasahara, A. Matsuo, Proc. Combust. Inst. 33 (2011) 2343-2349.

[16] S. Maeda, J. Kasahara, A. Matsuo, Combust. Flame 159 (2012) 887-896.

[17] Y. Kudo, Y. Nagura, J. Kasahara, Y. Sasamoto, A. Matsuo, Proc. Combust. Inst. 33 (2011) 2319-2326.

[18] H. Nakayama, T. Moriya, J. Kasahara, A. Matsuo, Y. Sasamoto, I. Funaki, Combust. Flame 159 (2012) 859-869.

[19] H. Nakayama, T. Moriya, J. Kasahara, A. Matsuo, Y. Sasamoto, I. Funaki, Propagation of Curved Detonation Waves Stabilized in Annular Channels with a Rectangular Cross-section, Trans. JSASS Aerospace Tech. Japan (in press).

[20] M. Kaneshige, J.E. Shepherd, Detonation Database, Technical Report FM97-8, GALCIT, 1997, available at http://www.galcitt.caltech.edu/detn_db/html/db.html.

[21] S. Gordon, B.J. McBride, Computer Program for Calculation of Complex Chemical Equilibrium Compositions and Applications, RP-1311, NASA, 1994.

[22] J.A. Sethian, Level Set Methods and Fast Marching Methods, Cambridge University Press, Cambridge, U.K., 1999, pp. 34-38. 


\section{Table title and figure captions}

Table 1 Experimental conditions (temperature is $298 \pm 6 \mathrm{~K}$ ).

Fig. 1. Geometric relationship in a curved detonation wave stabilized in a rectangular-cross-section curved channel.

Fig. 2. Schematics of curved channels.

Fig. 3. Relations between $r_{\mathrm{i}}$ and $\lambda$ in terms of the propagation mode.

Fig. 4. MSOP photograph of a curved detonation wave in the stable mode [19].

Fig. 5. Approximated front shock shape of a curved detonation wave in the stable mode $\left(\mathrm{C}_{2} \mathrm{H}_{4}+3 \mathrm{O}_{2}, \quad r_{\mathrm{i}}=20 \mathrm{~mm}, \lambda=0.70 \mathrm{~mm}, \quad D_{\mathrm{n}, \mathrm{asy}}=0.980 D_{\mathrm{CJ}}, \quad m=8.10, \quad D_{\mathrm{n}, \mathrm{i}}=0.87235 D_{\mathrm{CJ}}\right.$, $\left.\omega=1.0231 \times 10^{5} \mathrm{rad} / \mathrm{s}\right)$.

Fig. 6. Approximated $D_{\mathrm{n}}$ distribution of a curved detonation wave in the stable mode $\left(\mathrm{C}_{2} \mathrm{H}_{4}+3 \mathrm{O}_{2}, \quad r_{\mathrm{i}}=20 \mathrm{~mm}, \lambda=0.70 \mathrm{~mm}, \quad D_{\mathrm{n}, \mathrm{asy}}=0.980 D_{\mathrm{CJ}}, \quad m=8.10, \quad D_{\mathrm{n}, \mathrm{i}}=0.87235 D_{\mathrm{CJ}}\right.$, $\left.\omega=1.0231 \times 10^{5} \mathrm{rad} / \mathrm{s}\right)$.

Fig. 7. $D_{\mathrm{n}} / D_{\mathrm{CJ}}-\lambda \kappa$ relations of curved detonation waves in the stable mode $\left(\mathrm{C}_{2} \mathrm{H}_{4}+3 \mathrm{O}_{2}\right)$.

Fig. 8. Comparison of the $D_{\mathrm{n}} / D_{\mathrm{CJ}}-\lambda \kappa$ relations.

Fig. 9. Reconstruction of the front shock behavior of curved detonation waves with the overall $D_{\mathrm{n}} / D_{\mathrm{CJ}}-\lambda \kappa$ relation.

Fig. 10. Front shock evolution of curved detonation waves reconstructed with the overall $D_{\mathrm{n}} / D_{\mathrm{CJ}}-\lambda \kappa$ relation $\left(r_{\mathrm{i}} / \lambda=23\right.$ and $\left.r_{\mathrm{i}} / \lambda=50\right)$.

Fig. 11. Self-similarity of the front shock shapes of curved detonation waves with different $r_{\mathrm{i}}$ $\left(\mathrm{C}_{2} \mathrm{H}_{4}+3 \mathrm{O}_{2}, r_{\mathrm{i}} / \lambda=36.3\right)$. 
Proceedings of the Combustion Institute 34 (2013) 1939-1947

H. Nakayama, J. Kasahara, A. Matsuo, I. Funaki

Fig. 12. Self-similarity of the front shock shapes of curved detonation waves with different test gases $\left(r_{\mathrm{i}}=20 \mathrm{~mm}, r_{\mathrm{i}} / \lambda=27.5\right)$. 
Proceedings of the Combustion Institute 34 (2013) 1939-1947

H. Nakayama, J. Kasahara, A. Matsuo, I. Funaki

Table 1

\begin{tabular}{cccc}
\multicolumn{4}{c}{ Experimental conditions (temperature is $298 \pm 6 \mathrm{~K})}$. \\
\hline Test gas & $p_{0}(\mathrm{kPa})$ & $\lambda(\mathrm{mm})^{\mathrm{a}}$ & $D_{\mathrm{CJ}}(\mathrm{m} / \mathrm{s})^{\mathrm{b}}$ \\
\hline $\mathrm{C}_{2} \mathrm{H}_{4}+3 \mathrm{O}_{2}$ & $20-100$ & $0.40-2.41$ & $2296-2376$ \\
$2 \mathrm{H}_{2}+\mathrm{O}_{2}$ & $30-190$ & $0.73-4.82$ & $2772-2873$ \\
$2 \mathrm{C}_{2} \mathrm{H}_{2}+5 \mathrm{O}_{2}+7 \mathrm{Ar}$ & $15-150$ & $0.23-3.00$ & $1928-2033$ \\
\hline${ }^{\mathrm{a}}$ Detonation Database, Caltech [20] \\
$\mathrm{C}_{2} \mathrm{H}_{4}+3 \mathrm{O}_{2}: \lambda=72.020 p_{0}{ }^{-1.1270}(\mathrm{SD}: 18 \%)$ \\
$2 \mathrm{H}_{2}+\mathrm{O}_{2}: \lambda=157.15 p_{0}{ }^{-1.0242}(\mathrm{SD}: 22 \%)$ \\
$2 \mathrm{C}_{2} \mathrm{H}_{2}+5 \mathrm{O}_{2}+7 \mathrm{Ar}: \lambda=61.522 p_{0}{ }^{-1.1173}(\mathrm{SD}: 7 \%)$ \\
${ }^{\mathrm{b}} \mathrm{CEA}_{2}$ NASA [21]
\end{tabular}

$(11$ text lines +2 blanks $) \times 7.6$ words/line $\times 1$ column $=99$ words 
Proceedings of the Combustion Institute 34 (2013) 1939-1947

H. Nakayama, J. Kasahara, A. Matsuo, I. Funaki

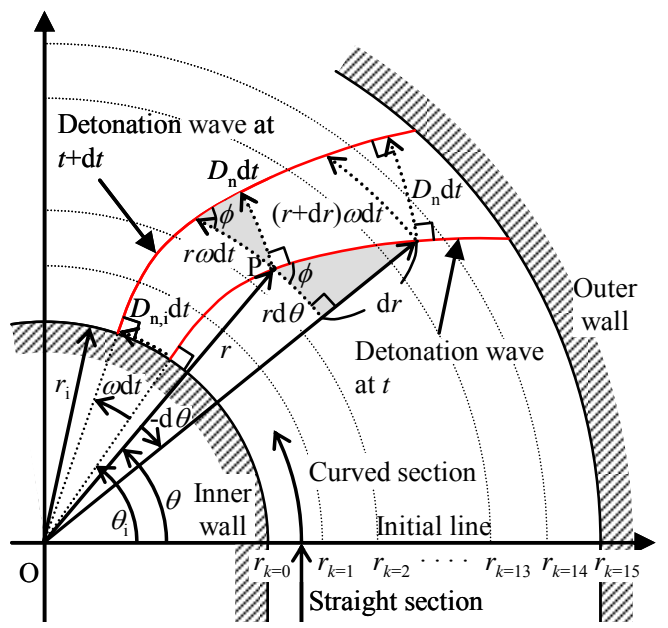

Fig. 1. Geometric relationship in a curved detonation wave stabilized in a rectangular-cross-section curved channel.

M1: $(62 \mathrm{~mm}+10 \mathrm{~mm}) \times 2.2$ words $/ \mathrm{mm} \times 1$ column +13 words $=171$ words 


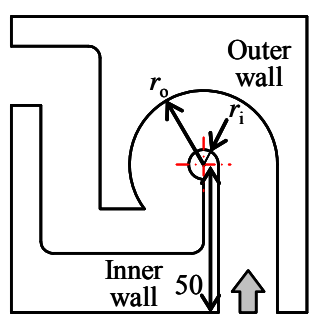

(1) $r_{\mathrm{i}}=5 \mathrm{~mm}, r_{\mathrm{o}}=25 \mathrm{~mm}$

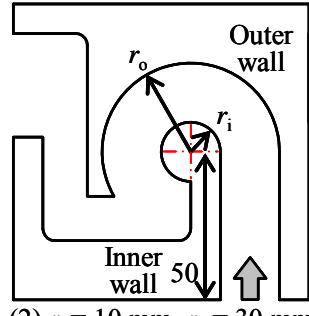

(2) $r_{\mathrm{i}}=10 \mathrm{~mm}, r_{\mathrm{o}}=30 \mathrm{~mm}$

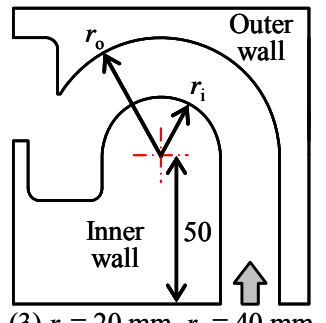

(3) $r_{\mathrm{i}}=20 \mathrm{~mm}, r_{\mathrm{o}}=40 \mathrm{~mm}$

Fig. 2. Schematics of curved channels.

M1: $(104 \mathrm{~mm}+10 \mathrm{~mm}) \times 2.2$ words $/ \mathrm{mm} \times 1$ column +4 words $=255$ words 


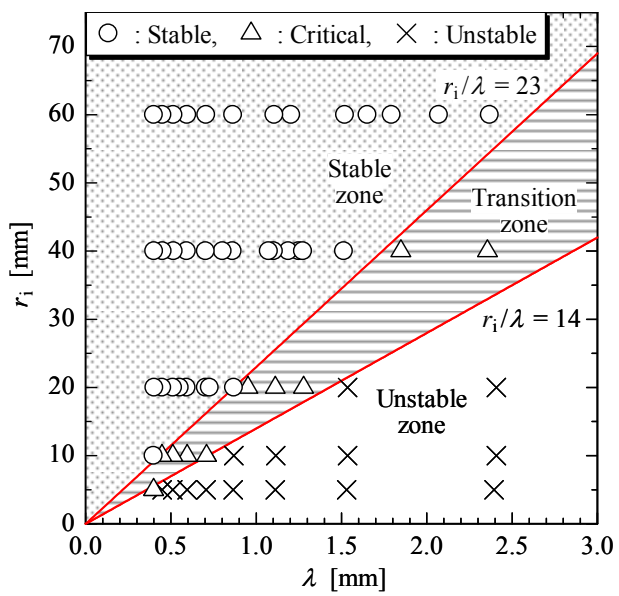

(a) $\mathrm{C}_{2} \mathrm{H}_{4}+3 \mathrm{O}_{2}$

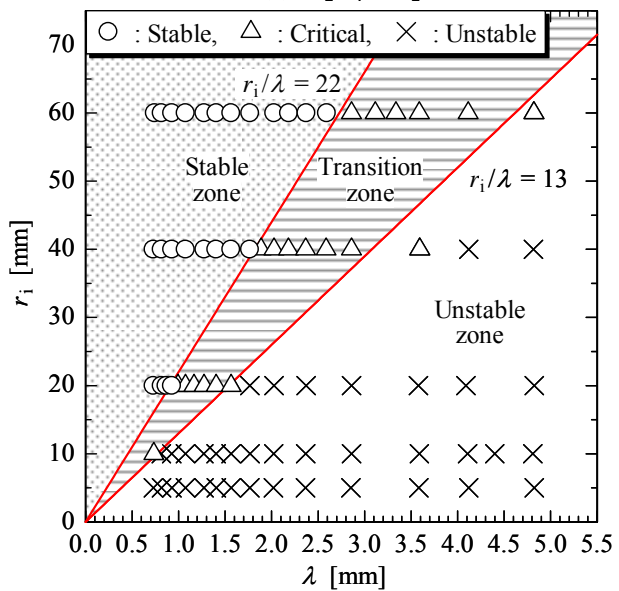

(b) $2 \mathrm{H}_{2}+\mathrm{O}_{2}$

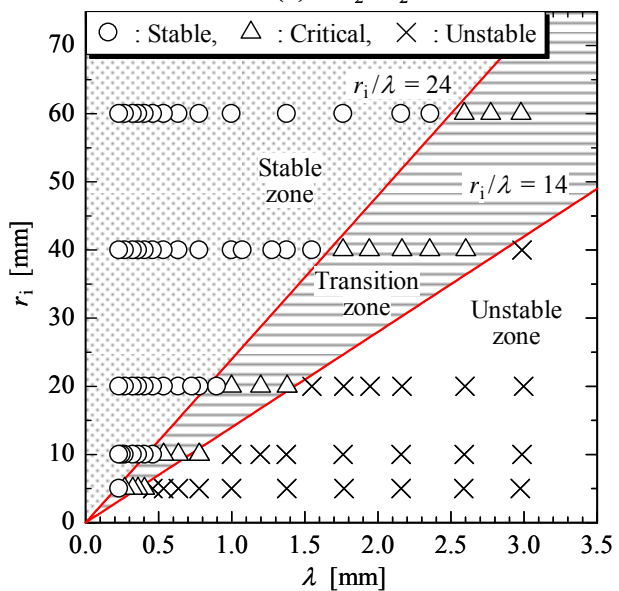

(c) $2 \mathrm{C}_{2} \mathrm{H}_{2}+5 \mathrm{O}_{2}+7 \mathrm{Ar}$

Fig. 3. Relations between $r_{\mathrm{i}}$ and $\lambda$ in terms of the propagation mode.

M1: $(189 \mathrm{~mm}+10 \mathrm{~mm}) \times 2.2$ words $/ \mathrm{mm} \times 1$ column +11 words $=449$ words 
Proceedings of the Combustion Institute 34 (2013) 1939-1947

H. Nakayama, J. Kasahara, A. Matsuo, I. Funaki

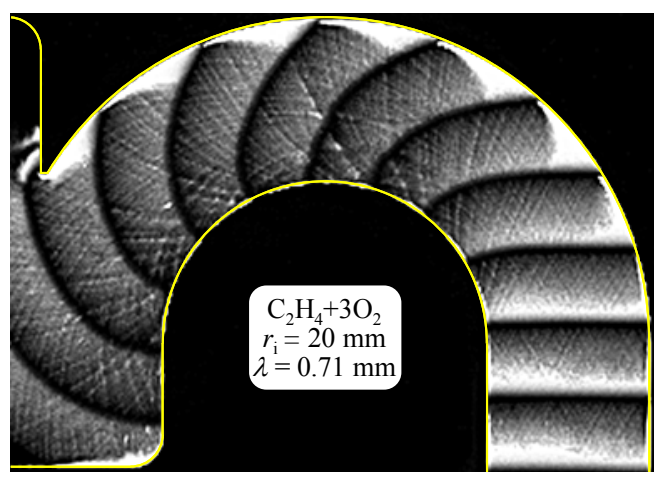

Fig. 4. MSOP photograph of a curved detonation wave in the stable mode [19].

M1: $(47 \mathrm{~mm}+10 \mathrm{~mm}) \times 2.2$ words $/ \mathrm{mm} \times 1$ column +12 words $=137$ words 
Proceedings of the Combustion Institute 34 (2013) 1939-1947

H. Nakayama, J. Kasahara, A. Matsuo, I. Funaki

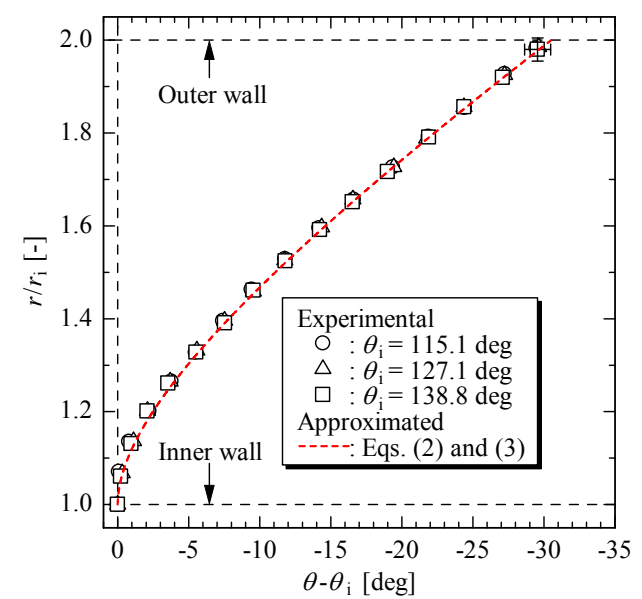

Fig. 5. Approximated front shock shape of a curved detonation wave in the stable mode $\left(\mathrm{C}_{2} \mathrm{H}_{4}+3 \mathrm{O}_{2}, r_{\mathrm{i}}=20 \mathrm{~mm}, \lambda=0.70 \mathrm{~mm}, D_{\mathrm{n}, \mathrm{asy}}=0.980 D_{\mathrm{CJ}}\right.$, $\left.m=8.10, D_{\mathrm{n}, \mathrm{i}}=0.87235 D_{\mathrm{CJ}}, \omega=1.0231 \times 10^{5} \mathrm{rad} / \mathrm{s}\right)$.

M1: $(59 \mathrm{~mm}+10 \mathrm{~mm}) \times 2.2$ words $/ \mathrm{mm} \times 1$ column +23 words $=175$ words 
Proceedings of the Combustion Institute 34 (2013) 1939-1947

H. Nakayama, J. Kasahara, A. Matsuo, I. Funaki

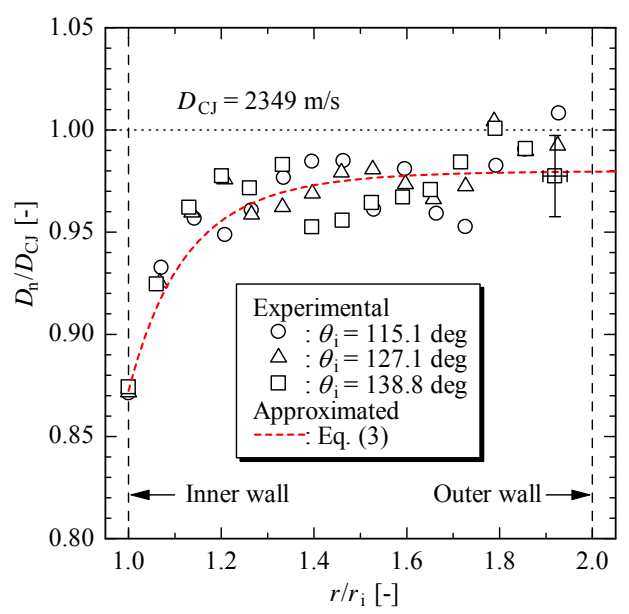

Fig. 6. Approximated $D_{\mathrm{n}}$ distribution of a curved detonation wave in the stable mode $\left(\mathrm{C}_{2} \mathrm{H}_{4}+3 \mathrm{O}_{2}, r_{\mathrm{i}}=20 \mathrm{~mm}, \lambda=0.70 \mathrm{~mm}, D_{\mathrm{n}, \mathrm{asy}}=0.980 D_{\mathrm{CJ}}\right.$, $\left.m=8.10, D_{\mathrm{n}, \mathrm{i}}=0.87235 D_{\mathrm{CJ}}, \omega=1.0231 \times 10^{5} \mathrm{rad} / \mathrm{s}\right)$.

M1: $(60 \mathrm{~mm}+10 \mathrm{~mm}) \times 2.2$ words $/ \mathrm{mm} \times 1$ column +22 words $=176$ words 
Proceedings of the Combustion Institute 34 (2013) 1939-1947

H. Nakayama, J. Kasahara, A. Matsuo, I. Funaki

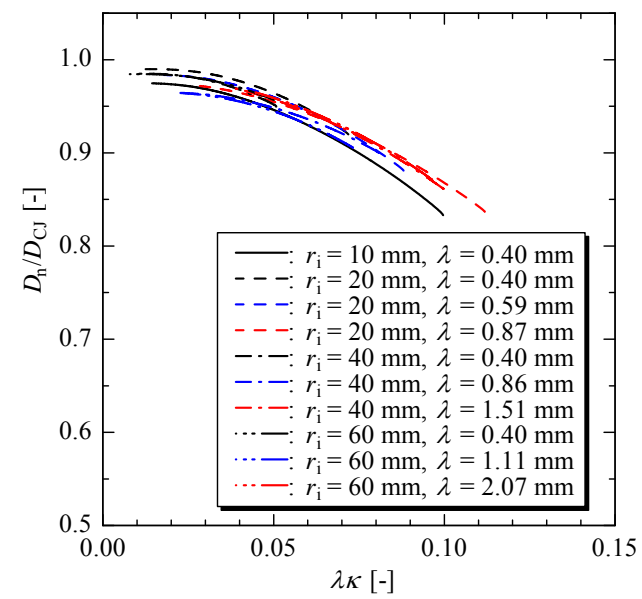

Fig. 7. $D_{\mathrm{n}} / D_{\mathrm{CJ}}-\lambda \kappa$ relations of curved detonation waves in the stable mode $\left(\mathrm{C}_{2} \mathrm{H}_{4}+3 \mathrm{O}_{2}\right)$.

M1: $(59 \mathrm{~mm}+10 \mathrm{~mm}) \times 2.2$ words $/ \mathrm{mm} \times 1$ column +11 words $=163$ words 
Proceedings of the Combustion Institute 34 (2013) 1939-1947

H. Nakayama, J. Kasahara, A. Matsuo, I. Funaki

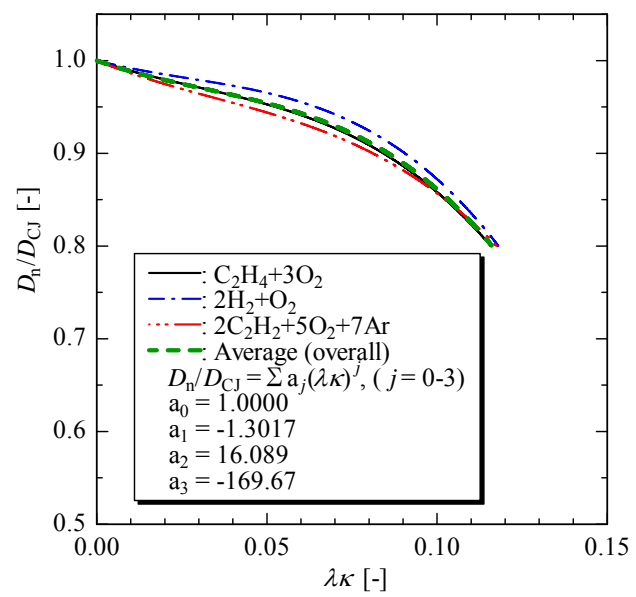

Fig. 8. Comparison of the $D_{\mathrm{n}} / D_{\mathrm{CJ}}-\lambda \kappa$ relations.

M1: $(59 \mathrm{~mm}+10 \mathrm{~mm}) \times 2.2$ words $/ \mathrm{mm} \times 1$ column +5 words $=157$ words 
Proceedings of the Combustion Institute 34 (2013) 1939-1947

H. Nakayama, J. Kasahara, A. Matsuo, I. Funaki

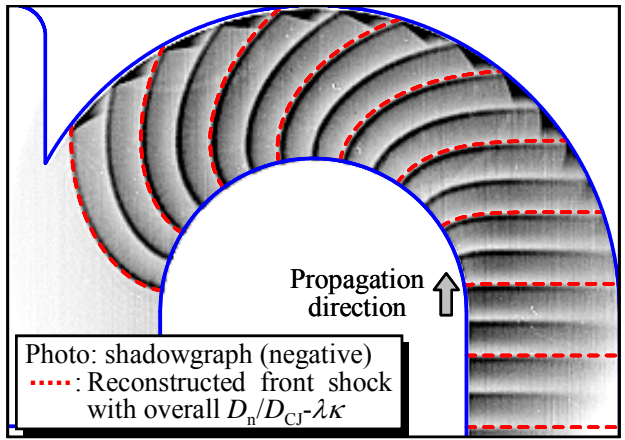

(a) $\mathrm{C}_{2} \mathrm{H}_{4}+3 \mathrm{O}_{2}, r_{\mathrm{i}}=20 \mathrm{~mm}, \lambda=0.70 \mathrm{~mm}$

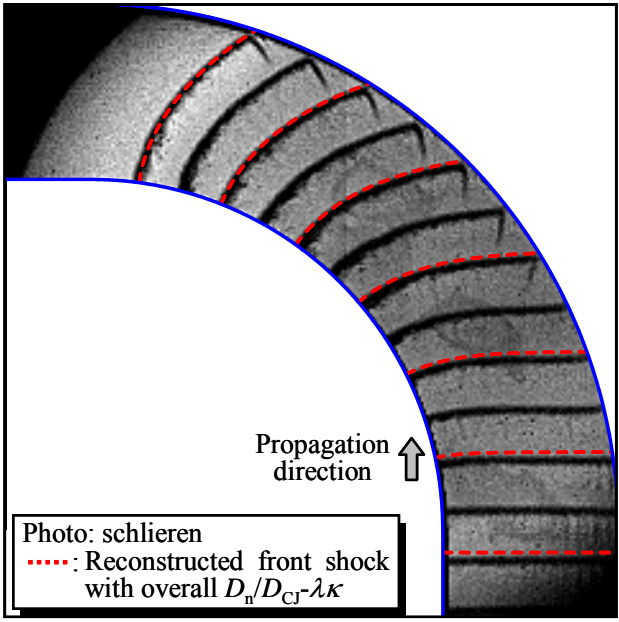

(b) $2 \mathrm{H}_{2}+\mathrm{O}_{2}, r_{\mathrm{i}}=40 \mathrm{~mm}, \lambda=1.56 \mathrm{~mm}$

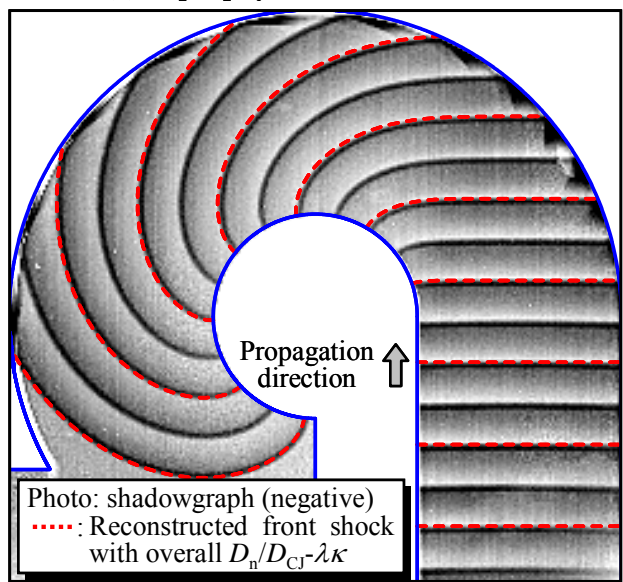

(c) $2 \mathrm{C}_{2} \mathrm{H}_{2}+5 \mathrm{O}_{2}+7 \mathrm{Ar}, r_{\mathrm{i}}=10 \mathrm{~mm}, \lambda=0.27 \mathrm{~mm}$

Fig. 9. Reconstruction of the front shock behavior of curved detonation waves with the overall $D_{\mathrm{n}} / D_{\mathrm{CJ}}-\lambda \kappa$ relation.

M1: $(177 \mathrm{~mm}+10 \mathrm{~mm}) \times 2.2$ words $/ \mathrm{mm} \times 1$ column +15 words $=426$ words 
Proceedings of the Combustion Institute 34 (2013) 1939-1947

H. Nakayama, J. Kasahara, A. Matsuo, I. Funaki

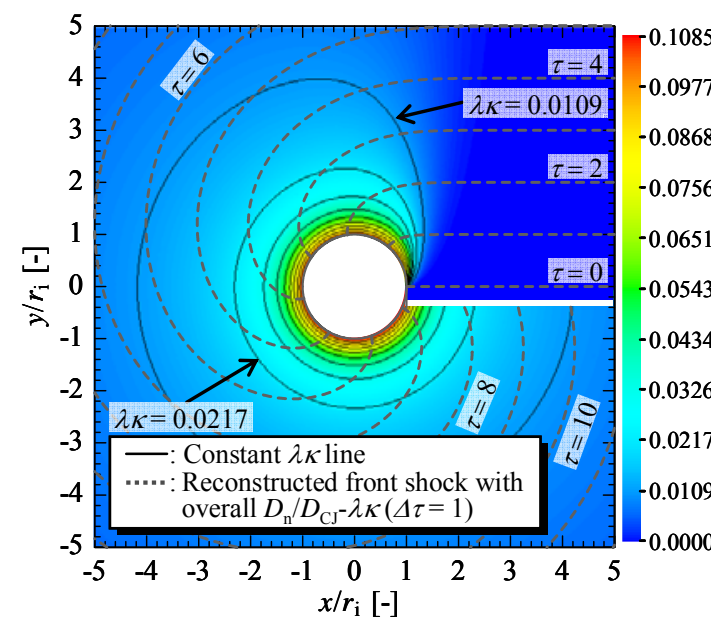

(a) Front shock evolution with $\lambda \kappa$ contour $\left(r_{\mathrm{i}} / \lambda=23\right)$

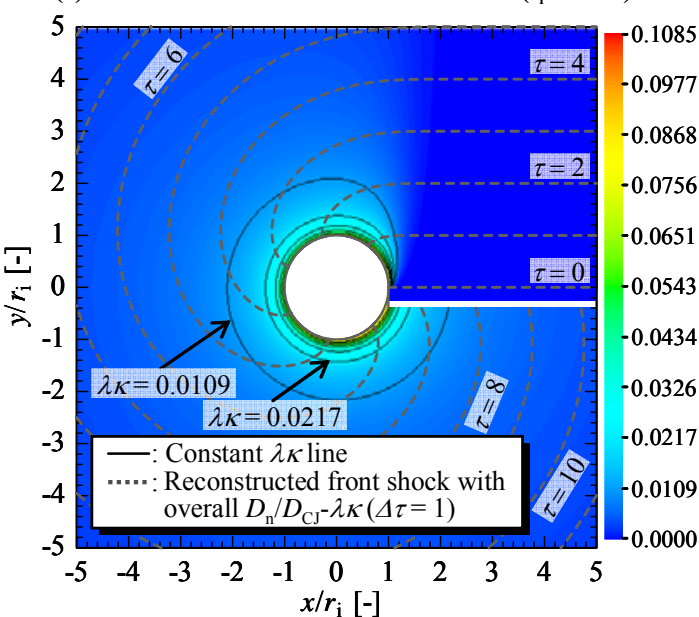

(c) Front shock evolution with $\lambda \kappa$ contour $\left(r_{\mathrm{i}} / \lambda=50\right)$

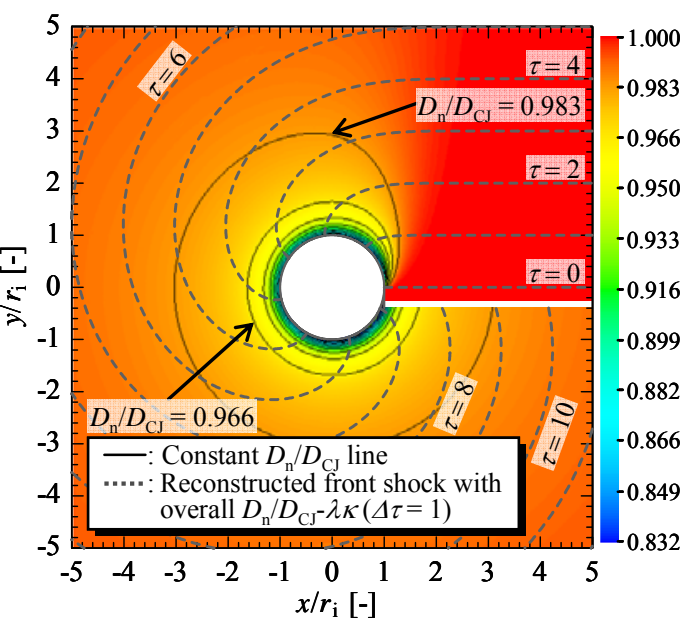

(b) Front shock evolution with $D_{\mathrm{n}} / D_{\mathrm{CJ}}$ contour $\left(r_{\mathrm{i}} / \lambda=23\right)$

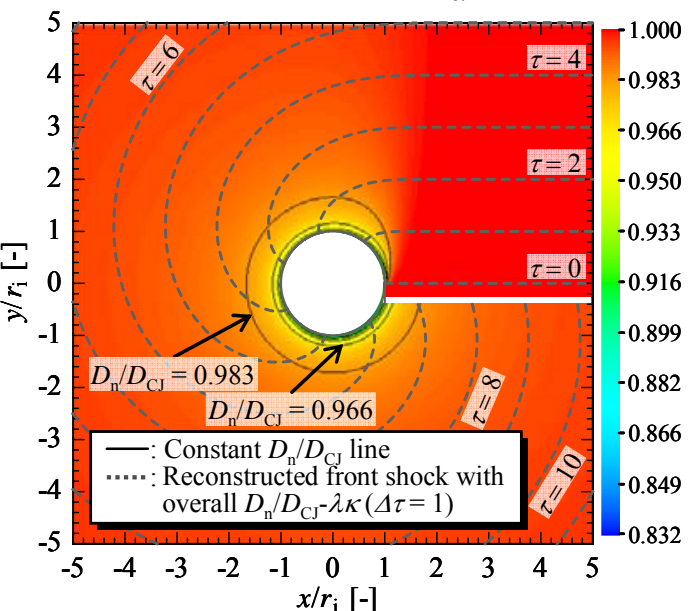

(d) Front shock evolution with $D_{\mathrm{n}} / D_{\mathrm{CJ}}$ contour $\left(r_{\mathrm{i}} / \lambda=50\right)$

Fig. 10. Front shock evolution of curved detonation waves reconstructed with the overall $D_{\mathrm{n}} / D_{\mathrm{CJ}}-\lambda \kappa$ relation $\left(r_{\mathrm{i}} / \lambda=23\right.$ and $\left.r_{\mathrm{i}} / \lambda=50\right)$.

M1: $(130 \mathrm{~mm}+10 \mathrm{~mm}) \times 2.2$ words $/ \mathrm{mm} \times 2$ column +16 words $=632$ words 
Proceedings of the Combustion Institute 34 (2013) 1939-1947

H. Nakayama, J. Kasahara, A. Matsuo, I. Funaki

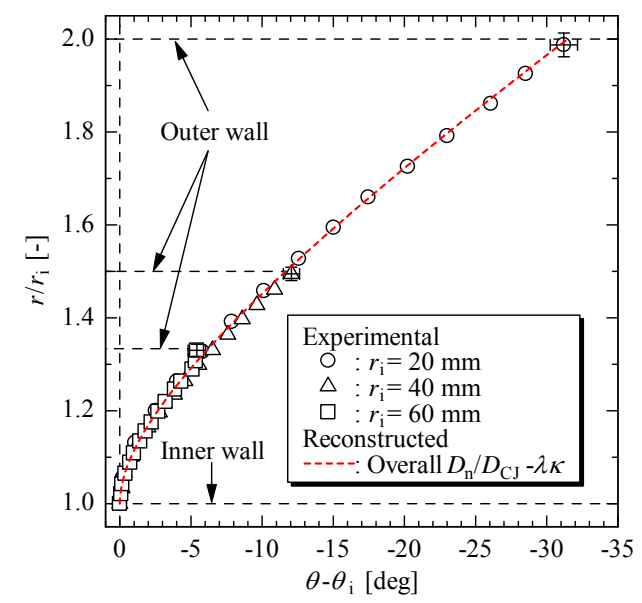

Fig. 11. Self-similarity of the front shock shapes of curved detonation waves with different $r_{\mathrm{i}}\left(\mathrm{C}_{2} \mathrm{H}_{4}+3 \mathrm{O}_{2}, r_{\mathrm{i}} / \lambda=36.3\right)$.

M1: $(59 \mathrm{~mm}+10 \mathrm{~mm}) \times 2.2$ words $/ \mathrm{mm} \times 1$ column +15 words $=167$ words 
Proceedings of the Combustion Institute 34 (2013) 1939-1947

H. Nakayama, J. Kasahara, A. Matsuo, I. Funaki

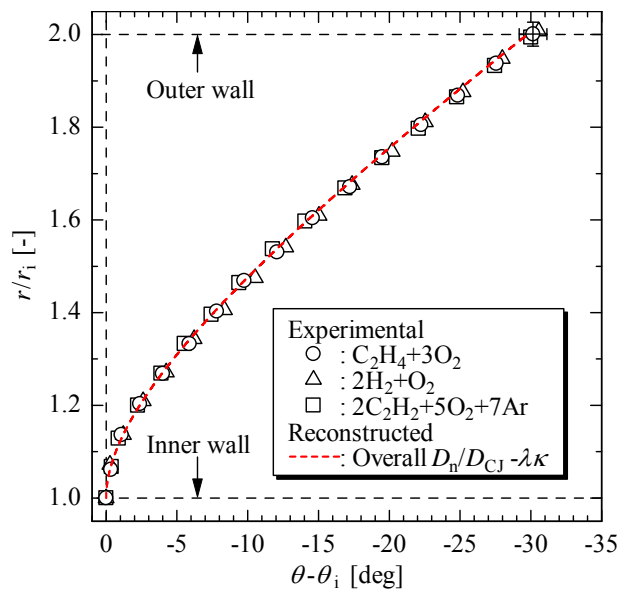

Fig. 12. Self-similarity of the front shock shapes of curved detonation waves with different test gases $\left(r_{\mathrm{i}}=20 \mathrm{~mm}, r_{\mathrm{i}} / \lambda=27.5\right)$.

M1: $(59 \mathrm{~mm}+10 \mathrm{~mm}) \times 2.2$ words $/ \mathrm{mm} \times 1$ column +17 words $=169$ words 